\title{
Multicentric reticulohistiocytosis with arthritis and cardiac infiltration: regression following treatment for underlying malignancy
}

\author{
C M Lambert, G Nuki
}

\begin{abstract}
The case is reported of a 63 year old man presenting with a rapidly destructive symmetrical polyarthritis and widespread papular nodular skin lesions, confirmed by a biopsy to be due to multicentric reticulohistiocytosis. Biventricular cardiac failure developed secondary to extensive myocardial infiltration with multicentric reticulohistiocytosis, a complication of this disease which has not previously been reported. The joint, skin, and cardiac manifestations of multicentric reticulohistiocytosis substantially regressed following resection of an associated squamous cell carcinoma. This report adds to the small amount of published work which suggests that multicentric reticulohistiocytosis can be a paraneoplastic disease that may respond to treatment directed at the underlying tumour.
\end{abstract}

Multicentric reticulohistiocytosis is an uncommon disease of unknown aetiology sufficiently similar in its presentation to rheumatoid arthritis for it often to be initially mistaken as such. Although the joints and skin are the most commonly affected sites, multicentric reticulohistiocytosis may become a systemic disease with a poor prognosis. ${ }^{2}$

\section{Case report}

A 63 year old previously healthy man presented with a three month history of polyarthralgia and morning stiffness affecting the hands, wrists, and knees together with an extensive painless, pruritic rash. Examination showed synovitis, bilateral knee effusions, and olecranon bursitis. Crops of red and brown papules $1-5 \mathrm{~mm}$ in diameter were present over the chest and abdomen. Xanthelasmata were noted but there were no other cutaneous features of hyperlipidaemia and the examination was otherwise normal.

The erythrocyte sedimentation rate was increased at $43 \mathrm{~mm}$ in the first hour but the full blood count, plasma urea and electrolytes, liver function tests, fasting lipids, immunoglobulins, and rheumatoid factor were all normal. Hand radiographs showed soft tissue swelling, periarticular osteoporosis, and loss of articular cartilage of the proximal and distal interphalangeal joints. The diagnosis of multicentric reticulohistiocytosis was established by histological examination of a papule, which showed infiltration with large mononuclear and multinuclear histiocytic cells with eosinophilic granular cytoplasm. The histiocytic cells contained periodic acid-Schiff positive material typical of this disease. The synovial fluid aspirate contained polymorphs, a few macrophages and lymphocytes, but there were no specific features of multicentric reticulohistiocytosis.

The patient was initially treated symptomatically with rest, non-steroidal anti-inflammatory drugs (NSAIDs) and intra-articular steroids. The synovitis persisted, however, and cyclophosphamide ( $15 \mathrm{mg} / \mathrm{kg} / \mathrm{month}$ ) was given by intermittent intravenous infusion. There was some improvement in joint symptoms and synovitis after two infusions but the skin lesions remained static.

Three months later the patient presented with dyspnoea at rest and signs of acute biventricular cardiac failure. Flexor tenosynovitis with deformities of the fingers had developed. The skin disease had progressed with the development of several larger nodules which were freely mobile within the subcutaneous tissue over the extensor aspect of his forearms and hands (fig 1). Further papules

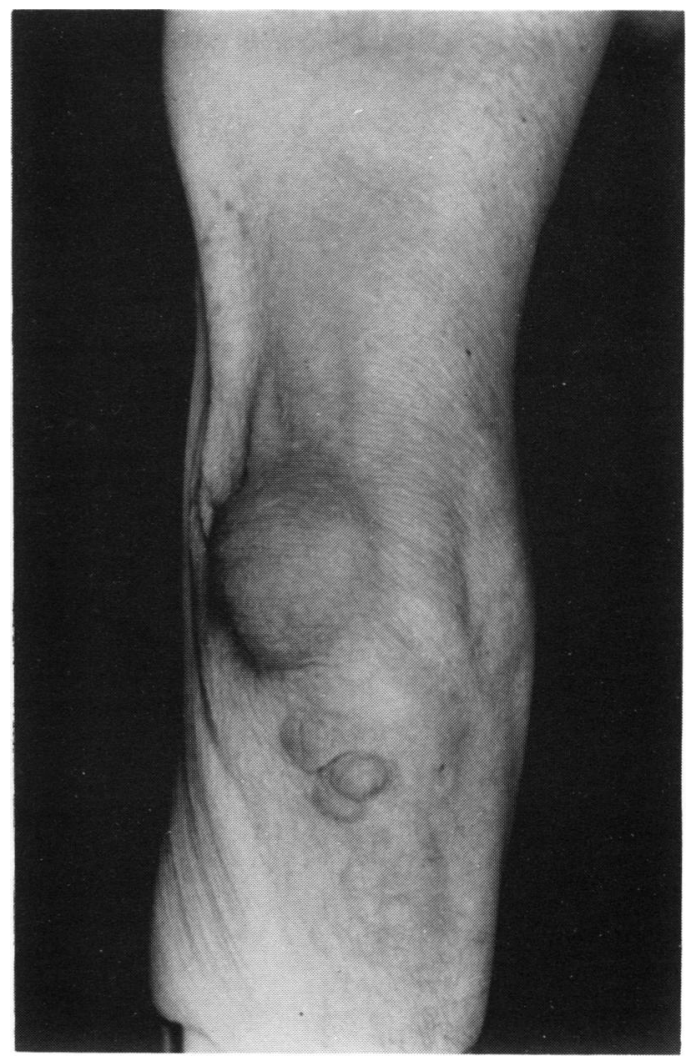

Figure 1 Right elbow with an olecranon bursitis and subcutaneous nodules. 
were present over the knees and periumbilical area (fig 2). A necrotic nodule on the glans penis had ulcerated and was tethered to the underlying tissue.

A 12 lead electrocardiogram showed loss of $r$ wave progression in the lateral chest leads but the changes did not evolve over the subsequent days and serial cardiac enzymes remained normal. A chest radiograph showed pulmonary oedema with cardiomegaly and pleural effusions. Echocardiography and magnetic resonance imaging showed that the impairment of ventricular function was due to widespread infiltration of the myocardium with multicentric reticulohistiocytosis. The arthritis had progressed radiologically to affect the right ulna styloid and intercarpal joints (fig 3). Biopsy of the penile lesion revealed a squamous cell carcinoma.

The cardiac failure was difficult to manage despite treatment with frusemide (up to $160 \mathrm{mg}$ daily) and the introduction of a vasodilator (isosorbide dinitrate $20 \mathrm{mg}$ twice daily). Subtotal penile amputation was, however, followed by a prompt improvement in the dyspnoea and oedema. In view of the risk posed by a general anaesthetic the procedure was performed using a spinal block with care to avoid overhydration after the operation.

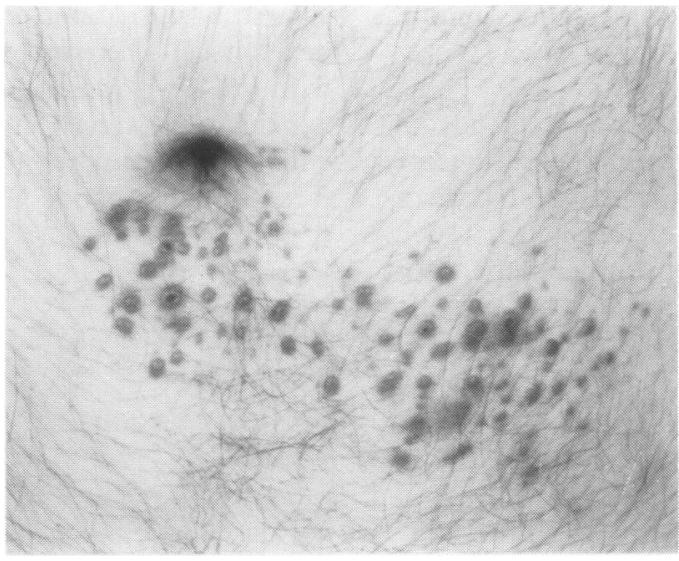

Figure 2 Periumbilical area with multiple papules.

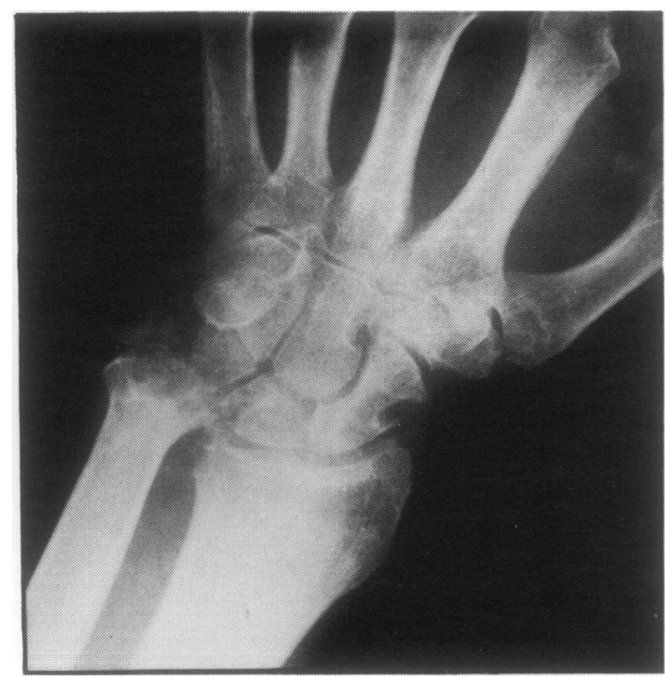

Figure 3 Radiograph showing erosions of the right carpus and ulna styloid.

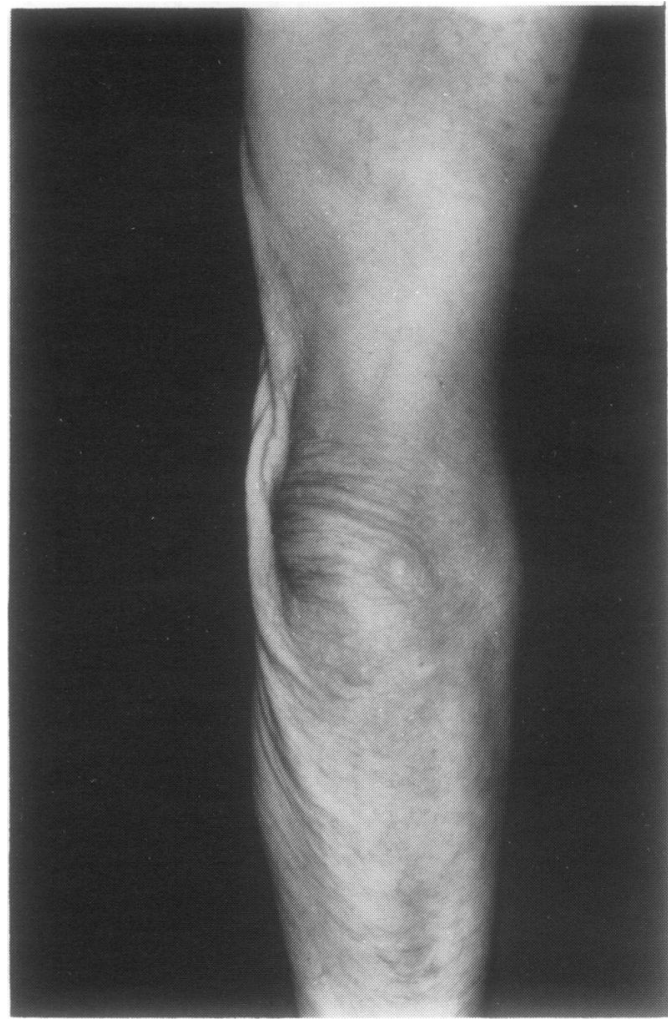

Figure 4 Regression of nodules following excision of an associated malignancy.

Frusemide alone (40 $\mathrm{mg}$ daily) was adequate to control his symptoms within a few days of excision. Four months after the operation there was no sign of cardiac failure and the cutaneous lesions had substantially regressed (fig 4 ). The heart size on a chest radiograph and the ventricular function at echocardiography were within normal limits. Repeat magnetic resonance imaging confirmed the improvement with a uniform signal intensity over most of the left ventricular wall and interventricular septum. There were no new erosions on hand radiographs.

One year after his original presentation the patient developed an inoperable local recurrence of a carcinoma in the penile stump and inguinal lymph nodes. There was no improvement in his condition following radiotherapy and chemotherapy and the patient died within a few weeks of developing pulmonary metastases. Despite recurrence of the tumour there was no sign of reactivation of the multicentric reticulohistiocytosis and in particular the patient had no further cardiac complications.

\section{Discussion}

The pathogenesis of multicentric reticulohistiocytosis remains obscure. Tissue damage and inflammation are thought to be mediated through the activation of histiocytes and the liberation of neutral proteases and acid hydrolases. ${ }^{3}$ The mechanisms for this are unknown but the suggestion that neoplastic cells may be implicated is supported by the improvement in multicentric reticulohistiocytosis concomitant with tumour regression and by the association in some reports of reactivation of multicentric reticulohistiocytosis with tumour recurrence. ${ }^{3}$ 
As this report illustrates, however, recurrence is not always heralded by a flare in the multicentric reticulohistiocytosis and so other factors must govern histiocytic activation in this disease, possibly by exerting an inhibitory effect.

Xanthelasmata and hypercholesterolaemia are present in $30 \%$ of patients with multicentric reticulohistiocytosis. ${ }^{45}$ The histiocytic accumulation of phospholipids, neutral fats, and lipoid polypeptide material is a prominent histological feature of multicentric reticulohistiocytosis and implies an abnormality of lipid handling in this disease. The varied nature of the intracellular accumulation, however, is more in favour of this being a secondary phenomenon. ${ }^{6}$

There is a recognised association between multicentric reticulohistiocytosis and a number of other diseases including neoplasms $(28 \%)$, hypothyroidism $(6 \%)$, diabetes $(6 \%)$, tuberculosis $(6 \%)$, Sjögren's syndrome, and primary biliary cirrhosis. ${ }^{6} 7$ In this respect a wide spectrum of neoplasms have been identified, including haematological and solid malignancies. $^{7}$ Multicentric reticulohistiocytosis precedes the diagnosis of the malignancy in $75 \%$ of patients, sometimes by up to ten months. ${ }^{3}$ In contrast to multicentric reticulohistiocytosis without an associated neoplasm, in which most patients present with arthritis, $90 \%$ of those with such an association present with skin lesions as either the only manifestation $(45 \%)$ or together with an arthropathy $(45 \%) .^{3}$

The arthritis of multicentric reticulohistiocytosis is usually symmetrical and erosive with a tendency to affect the distal and the proximal interphalangeal joints, distinguishing it from rheumatoid arthritis. ${ }^{1}$ Progression to arthritis mutilans with subarticular bone resorption occurs in $45 \%$ of patients. ${ }^{8}$ The cervical spine may be affected, leading to atlanto-axial instability. ${ }^{9}$ The arthritis is usually self limiting and 'burns out' after several years leaving mechanical deformities. ${ }^{10}$

The radiographic features of multicentric reticulohistiocytosis are similar to those of rheumatoid arthritis with well circumscribed articular erosions. The joint space narrowing and periarticular osteoporosis may be less than expected for the degree of erosion. Isotope scanning may be helpful in detecting occult systemic disease as gallium concentrates in these granulomatous deposits. ${ }^{11} 12$

The cardiopulmonary complications of multicentric reticulohistiocytosis are rare and, as far as we are aware, this is the first report to describe extensive myocardial infiltration with this disease. Magnetic resonance imaging provided a sensitive and non-invasive method of monitoring this manifestation of the disease. Pulmonary infiltrates were reported in $20 \%$ of patients in one study ${ }^{5}$ and pleural effusions, pulmonary infarction, endocardial damage, and fibrinous pericarditis are other documented cardiopulmonary complications which carry a high death rate. ${ }^{13}$

It is difficult to evaluate the efficacy of treatment for multicentric reticulohistiocytosis owing to the rarity of the disease and the tendency for spontaneous remission. Trials with lipid lowering drugs, steroids, azathioprine, and isoniazid have not shown consistent benefit. ${ }^{6}$ There is reasonable evidence that cytotoxic drugs, particularly alkylating drugs, may modify the disease. ${ }^{6} \mathrm{~A}$ positive tuberculin test is seen in $50 \%$ of patients with multicentric reticulohistiocytosis, but active tuberculosis is rare. One case has been reported in which complete resolution of multicentric reticulohistiocytosis followed treatment for tuberculosis. ${ }^{14}$ In patients with an associated malignancy the multicentric reticulohistiocytosis has responded to treatment for the neoplasm either by excision, endocrine manipulation, or chemotherapy. ${ }^{3}$

This paper reports the regression of multicentric reticulohistiocytosis affecting the major organs following tumour resection and highlights the importance of recognising such an association.

Melton J W, Irby $\mathrm{R}$ Multicentric reticulohistiocytosis. Arthritis Rheum 1972; 15: 221-6.

2 Fast A. Cardiopulmonary complications in multicentric reticulohistiocytosis. Arch Dermatol 1976; 112: 1139-41.

3 Nunnick J C, Krusinski P A, Yates J W. Multicentric reticulohistiocytosis and cancer: a case report and review of the literature. Med Pediatr Oncol 1985; 13: 273-9.

4 Barrow M V, Holubar K. Multicentric reticulohistiocytosis. A review of 33 patients. Medicine (Baltimore) 1969; 48: 287-305.

5 Orkin M, Goltz R W, Good R A, et al. A study of multicentric reticulohistiocytosis. Arch Dermatol 1964; 89: 640-54

6 Doherty M, Martin M F R, Dieppe P A. Multicentric reticulohistiocytosis associated with primary biliary cirrhosis: successful treatment with cytotoxic agents. Arthritis Rheum 1984; 27: 344-8

7 Catterall M D. Multicentric reticulohistiocytosis: a review of eight cases. Clin Exp Dermatol 1980; 5: 267-9.

8 Bortz A I, Vincent M. Lipoid dermatoarthrosis and arthritis mutilans. Am f Med 1961; 30: 951-60.

9 Martel W, Abell M R, Duff I F. Cervical spine involvement in lipoid dermatoarthrosis. Radiology 1961; 77: 613-7.

10 Hanauer L B. Reticulohistiocytosis: remission after cyclophosphamide therapy. Arthritis Rheum 1972; 15: 636-40.

11 Gold R H, Metzger A L, Mirra J M. Multicentric reticulohistiocytosis, an erosive polyarthritis with distinctive clinical and roentographic and pathologic features. $A m \mathcal{F}$ Roentgenol 1975; 124: 610-24.

12 Flurey N, Dimauro J, Eng A, et al. Multicentric reticulohistiocytosis with salivary gland involvement and pericardial histiocytosis with salivary gland involvement and

13 Warin R P, Evans C D, Hewitt M, et al. Reticulohistiocytosis (lipoid dermatarthrosis). BM J 1957; i: 1387-91.

14 Gold K D, Sharp J T, Estrada R E, et al. Relationship between multicentric reticulohistiocytosis and tuberculosis. JAMA 1977; 237: 2213-4. 